\title{
Hot STM of nanostructure dynamics on $\mathrm{SrTiO}_{3}(001)$
}

\author{
H L Marsh, D S Deak, F Silly, A I Kirkland and M R Castell \\ Department of Materials, University of Oxford, Parks Road, Oxford OX1 3PH, UK \\ E-mail: martin.castell@materials.ox.ac.uk
}

Received 7 April 2006, in final form 23 May 2006

Published 20 June 2006

Online at stacks.iop.org/Nano/17/3543

\begin{abstract}
The dynamics of nanostructured surface phases on $\mathrm{SrTiO}_{3}(001)$ have been analysed using in situ scanning tunnelling microscopy (STM) above $800^{\circ} \mathrm{C}$. During high-temperature annealing, the formation, growth and ordering of the nanostructures has been observed. Dilines, with a width of $\sim 1 \mathrm{~nm}$, are formed from a $\mathrm{TiO}_{2}$-rich intermediary at $800^{\circ} \mathrm{C}$. STM during annealing at $825^{\circ} \mathrm{C}$ has enabled us to follow both the growth and dissolution of dilines. Following extended annealing, trilines with a width of $\sim 2 \mathrm{~nm}$ and ordered two-dimensional (2D) nano-arrays form from the diline domains. Our observations of diline dissolution implies random nucleation and growth, followed by rearrangement at elevated temperature to form domains.
\end{abstract}

M This article features online multimedia enhancements

\section{Introduction}

Self-assembled nanometre-sized structures are of particular interest due to their importance in the development of novel electronic devices such as single electron transistors and quantum dot lasers [1, 2]. Oxide crystal surfaces, such as $\mathrm{SrTiO}_{3}$, are good candidates for studying the growth of nanostructures as these are electrically insulating. Nanostructures grown on these materials may find application in the development of arrays of nanowires and quantum dots [3].

In general, nanostructures tend to form at elevated temperatures, but are often observed, using scanning tunnelling microscopy (STM), at room temperature [4-6]. This approach provides many insights into their properties, but does not show direct evidence of their nucleation and growth behaviour. Therefore, in order to study nucleation and growth directly, hot STM imaging is needed. However, obtaining STM images is more challenging at high temperatures due to sample, stage and tip drift arising from the temperature gradient. These experimental difficulties notwithstanding, Si(111) has been imaged at temperatures of up to $920^{\circ} \mathrm{C}$ in order to study surface reconstructions [7]. Recently, there have been hot STM studies of $\mathrm{GdSi}_{2}$ nanowire reactions on $\mathrm{Si}(100)$ which have helped to define the structure of a new type of nanowire [8]. Hot STM imaging has also been used to identify an intermediate, Bi-related, linear structure formed during $\mathrm{Bi} / \mathrm{Si}(001)$ surface annealing [9].
STM of $\mathrm{SrTiO}_{3}(001)$ has been carried out at temperatures of just under $800^{\circ} \mathrm{C}$ and has been used to study the motion of step edges of the $(2 \times 2)$ surface reconstruction [10]. However, no study above the nanostructure formation temperature $\left(800^{\circ} \mathrm{C}\right)$ has been carried out, and hence no direct investigation of the nucleation and growth of nanostructures on $\mathrm{SrTiO}_{3}$ has yet been undertaken.

Nanostructures on the $\mathrm{c}(4 \times 2)$ reconstruction of $\mathrm{SrTiO}_{3}$ (001) were first described by Castell and several different variants have since been reported $[6,11,12]$. These structures have all been shown to be $\mathrm{TiO}_{2}$ rich using Auger electron spectroscopy [12].

The first nanostructures observed were dilines, which were initially referred to as nanolines [6] but, in order to distinguish them from the other types of structure, they have been renamed dilines [12]. Dilines, $\sim 1 \mathrm{~nm}$ in width and $0.2 \mathrm{~nm}$ in height, consist of two rows of protruding atoms or atomic complexes which have a period of $0.8 \mathrm{~nm}$. They form after an $\mathrm{Ar}^{+}$sputter and $30 \mathrm{~min} \mathrm{UHV}$ anneal at $825^{\circ} \mathrm{C}$. After a $30 \mathrm{~min}$ anneal at $850^{\circ} \mathrm{C}$, large domains of dilines with a $(6 \times 2)$ surface pattern form.

Trilines, with a width of $\sim 2 \mathrm{~nm}$ and consisting of three rows, form after $\mathrm{Ar}^{+}$sputtering and a 30 min anneal at higher temperature. The two outer rows are similar to those that make up a diline, however the central backbone has a shorter period of $0.4 \mathrm{~nm}$. The apparent height of the backbone of a triline varies with changing imaging bias. This is an electronic structure effect due to the difference in the local density of 
states between the backbone and the outer lines of the triline. Domains of trilines have a $(9 \times 2)$ symmetry.

It is also possible to form nanostructures that consist of $2 \mathrm{D}$ rectangular arrays. One of these is the waffle structure which has a surface periodicity of $(6 \times 8)$. This forms after repeated annealing of dilines and consists of domains of ordered trenches with a depth of $0.2 \mathrm{~nm}$ interspersed with $0.1 \mathrm{~nm}$ high cross-like structures.

To date, all STM observations of $\mathrm{SrTiO}_{3}$ nanostructures have been carried out at room temperature after an anneal at high temperature. From these experiments, dilines are known to form at around $825^{\circ} \mathrm{C}$, but their nucleation has never been observed directly. In this paper we analyse the nucleation and growth of nanostructures on $\mathrm{SrTiO}_{3}$ by observing their behaviour at temperatures up to $850{ }^{\circ} \mathrm{C}$ and accordingly investigate the mechanisms by which domain formation occurs.

\section{Experimental methods}

In bulk $\mathrm{SrTiO}_{3}$ a cubic perovskite structure $(a=0.3905 \mathrm{~nm})$ forms, and along a $\langle 001\rangle$ direction the crystal is made up of alternating $\mathrm{TiO}_{2}$ and $\mathrm{SrO}$ layers. Stoichiometric $\mathrm{SrTiO}_{3}$ is an insulator with a band gap of $3.2 \mathrm{eV}$, which becomes n-type semiconducting on doping with substitutional $\mathrm{Nb}^{5+}$ on the Ti sites. This increases the level of electrical conductivity, which enables analysis of the $\mathrm{SrTiO}_{3}(001)$ surface using STM.

Single crystals of $\mathrm{Nb}(0.5 \mathrm{wt} \%)$ doped $\mathrm{SrTiO}_{3}$ with epipolished (001) surfaces were supplied by PI-KEM (UK). A low room-temperature resistivity of $10^{-3} \Omega \mathrm{m}$, which increased with increasing sample temperature, was measured as a result of the high level of niobium doping. For STM imaging, a JEOL JSTM 4500XT was used with an ultrahigh-vacuum (UHV) chamber pressure of $10^{-8} \mathrm{~Pa}$. Chemically etched tungsten tips were used to obtain constant current images with a bias voltage applied to the sample. The sample was resistively heated in the UHV chamber by passing a current through it. A disappearing filament optical pyrometer was used to measure temperatures above $750{ }^{\circ} \mathrm{C}$. Initially, the samples were $\mathrm{Ar}^{+}$ion sputtered for 10 min with an ion energy of $0.5 \mathrm{keV}$ and an ion current of $7 \mu \mathrm{A}$.

\section{Results}

\subsection{Nucleation and growth of dilines}

After $\mathrm{Ar}^{+}$sputtering and annealing at $800^{\circ} \mathrm{C}$ for over $6 \mathrm{~h}$, dilines are observed to nucleate on the underlying $\mathrm{c}(4 \times 2)$ surface reconstruction (figure 1).

Figures $1(\mathrm{a})-(\mathrm{d})$ were taken at $800^{\circ} \mathrm{C}$ over a period of $24 \mathrm{~min}$. In these images, the bright pairs of rows are dilines and the dark area is a section of lower terrace. The shape of this area changes during observation as the upper terrace grows over it. A room-temperature image of dilines at the same scale is shown in figure 1(e). Although the characteristic pairs of dots are clearly visible at room temperature, they are not resolved in the hot images. However, a dark streak along the centre of the dilines distinguishes them from the other small atomic protrusions present at $800^{\circ} \mathrm{C}$.

In figure 1(a), the indicated ring surrounds a small atomic protrusion which acts as a nucleation centre. After 9 min
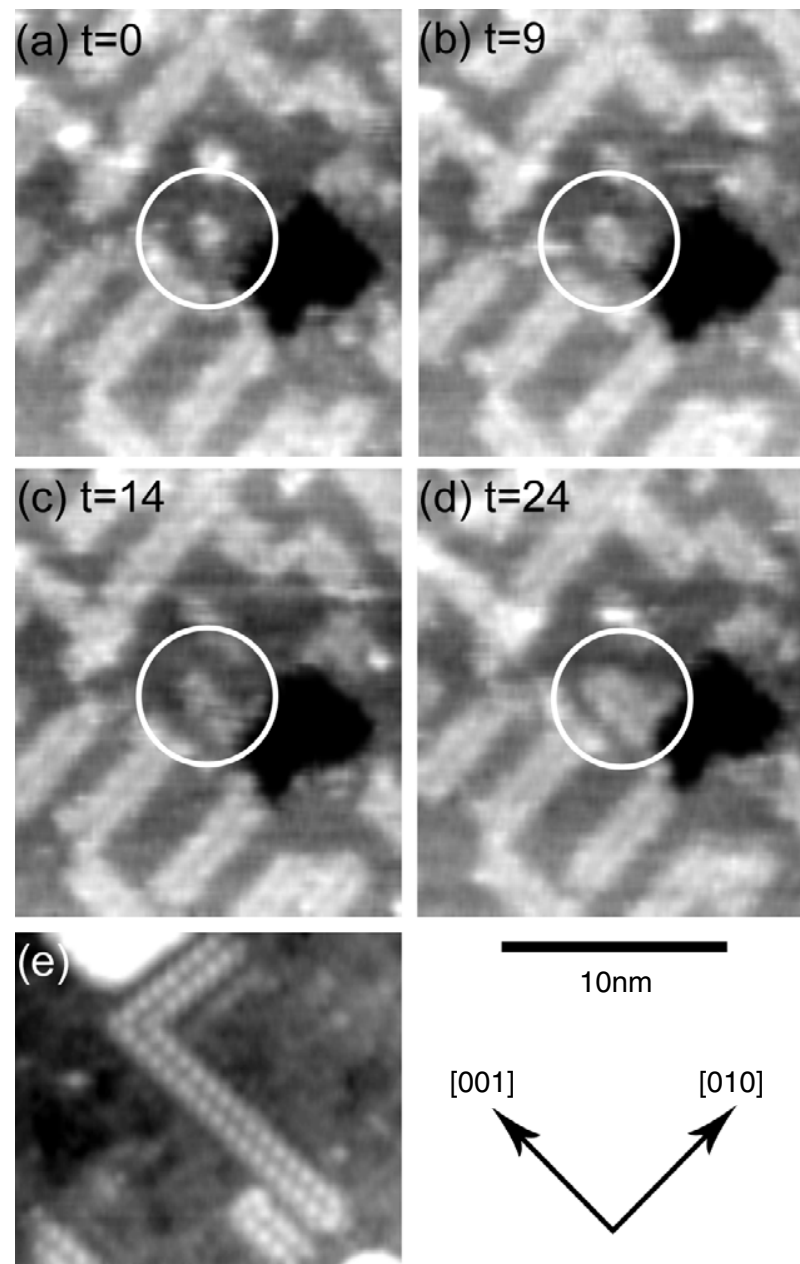

$10 \mathrm{~nm}$

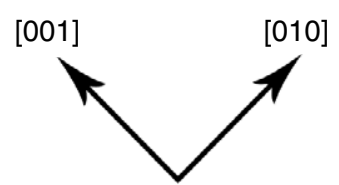

Figure 1. Formation of a diline from a nucleation centre at $800{ }^{\circ} \mathrm{C}$ (a)-(d). Time, $t$, is measured in minutes. (a) A nucleation centre is observed within the marked ring. (b) and (c) Growth of the nucleation centre is visible. (d) A fully formed nanoline is visible. (e) A room-temperature image of dilines. Sample voltages and tunnelling currents used: (a)-(d) $2.00 \mathrm{~V}, 0.10 \mathrm{nA}$; (e) $1.10 \mathrm{~V}$ and $0.10 \mathrm{nA}$.

(figure 1(b)), this has increased in size, but is less prominent than any diline. By figure 1(c), 5 min later, the structure is clearly elongated in one direction, but is still narrower and less bright than the established dilines. At 24 min (figure 1(d)), the nucleation centre is now of similar brightness to the surrounding dilines and has the distinct darker centre.

\subsection{Formation of diline domains}

After $\mathrm{Ar}^{+}$sputtering and annealing for $24 \mathrm{~h}$ at $825^{\circ} \mathrm{C}$, small domains of dilines begin to form. Large ordered domains are seen once the annealing temperature reaches $850^{\circ} \mathrm{C}$. Figure 2 shows room-temperature examples of randomly nucleated dilines (figure 2(a)) and larger domains of dilines (figure 2(b)). Hot STM experiments were therefore performed in order to eludidate the mechanisms by which the random isolated dilines rearrange into large ordered domains.

Figure 3 shows examples of the changes observed on the $\mathrm{SrTiO}_{3}(001)$ surface after over $24 \mathrm{~h}$ of annealing at $825^{\circ} \mathrm{C}$. In figure 3 , the darker areas are patches of lower terrace, 
Hot STM of nanostructure dynamics on $\mathrm{SrTiO}_{3}(001)$
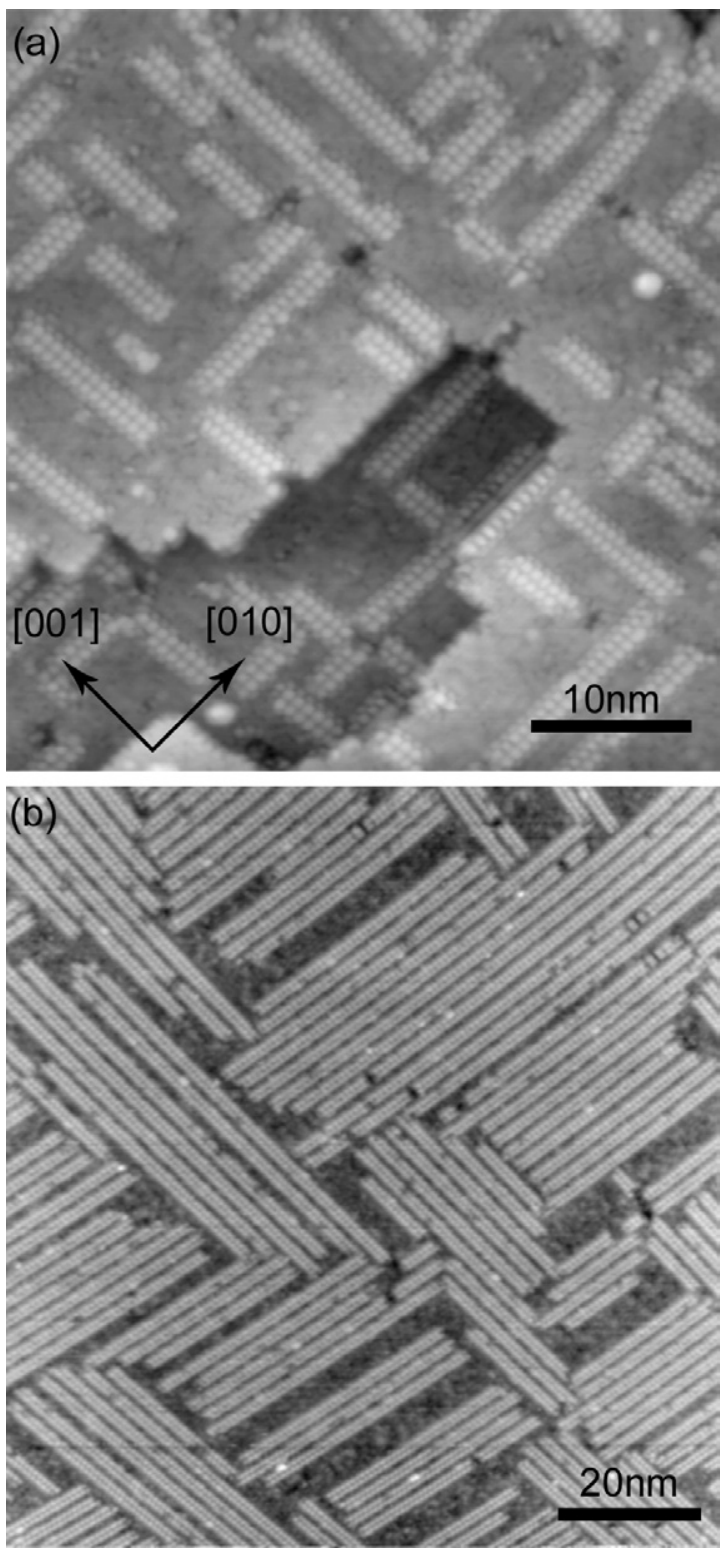

Figure 2. (a) Room-temperature image of randomly distributed dilines. (b) Larger domains of dilines at room temperature. Sample voltage and tunnelling current used: (a) and (b) $1.00 \mathrm{~V}, 0.07 \mathrm{nA}$.

and the growing nanolines are seen in the upper marked ring. Figure 3(a) shows the starting length of the nanolines and figures 3(b)-(d) show the growth of the upper terrace over the lower terrace. In figure 3(e), after $35 \mathrm{~min}$ the nanoline above the terrace has grown onto a new area of the terrace. During the course of the next three images $(20 \mathrm{~min}$ ) it is also observed to shrink and regrow.

In the lower marked ring, a nanoline disappears over the course of the scans. In figures 3(a)-(e), the nanoline shrinks progressively by one pair of atomic protrusions in each frame. In figure 3(f), a nanoline perpendicular to the original one can also be observed growing into the new gap. Seven minutes later (figure 3(g)) it has elongated, and after a further $11 \mathrm{~min}$ (figure 3(h)) it has extended to fill the space available.

In all our experiments, the nanolines were never observed to nucleate next to other nanolines. However, nanolines were observed in pairs, and these pairs were more stable than the single nanolines.

\subsection{Formation of trilines}

Once dilines begin to form domains and collect together in groups of two or three, then trilines are also observed. These form at a temperature of at least $825^{\circ} \mathrm{C}$ after annealing for over two days. The trilines were usually observed to form from a pair of dilines and were never seen to form from a single diline. They appear to be most stable, and grow to the greatest length when bounded on both sides, either by dilines or by the edge of a terrace or another domain.

The formation and growth of a triline at $850^{\circ} \mathrm{C}$ is shown in figures 4(a)-(d) over a period of $4 \mathrm{~min}$. Figure 4(e) shows a room-temperature image of trilines and dilines. The three rows of the triline are very clear at room temperature. At higher temperature, the triline is a wider and brighter line than the diline, but the individual rows are not visible. A small domain of trilines is present on the right-hand side of the image. In order to confirm that these structures were trilines, we measured the periodicity across the triline domains relative to that across the diline domains. The ratio of the periodicity across the rows of trilines to dilines at high temperature is 1.5. This is the same as the ratio measured at room temperature, thus verifying that the brighter nanolines are trilines.

In figure 4(a), two adjacent dilines are marked. In figure 4(b), recorded $1 \mathrm{~min}$ later, a short triline has nucleated from this diline. This triline grows (figure 4(c)) and then disappears again $2 \mathrm{~min}$ after it formed (figure 4(d)).

\subsection{Formation of waffle structures}

Waffle structures have also been observed at above $825^{\circ} \mathrm{C}$ after annealing times in excess of four days. Figures 5(a)-(d) show waffle structures forming and growing from dilines at $850^{\circ} \mathrm{C}$, over $37 \mathrm{~min}$. Figure 5(a) shows a small domain of dilines with a few elements of waffle structure visible within it. The number of waffle elements increases during the next $22 \mathrm{~min}$ (figure 5(b)) and, subsequently (figure 5(c)), the elements have joined together to form an extended section of waffle structure. After a further 12 min (figure 5(d)), a large area of the waffle structure is visible. This surface displays significant mobility, and the trenches and crosses that characterize it do not settle in any particular place.

Figure 5(e) is a room-temperature image with a large area of waffle structure in the bottom left, in which the characteristic dark trenches and lighter cross-like structures are visible. In the same image we also observe on the upper terrace a diline transforming into a waffle structure along its length.

\section{Discussion}

Dilines are observed to form via small nucleation centres. This nucleation structure is believed to be $\mathrm{TiO}_{2}$-rich as it produces the $\mathrm{TiO}_{2}$-rich dilines, as confirmed by previous Auger electron spectroscopy studies [12]. We also note that the dilines form with a specific length, of three or more pairs of atomic protrusions, within one STM scan, and then remain stable. In our high-temperature experiments, dilines are never observed 

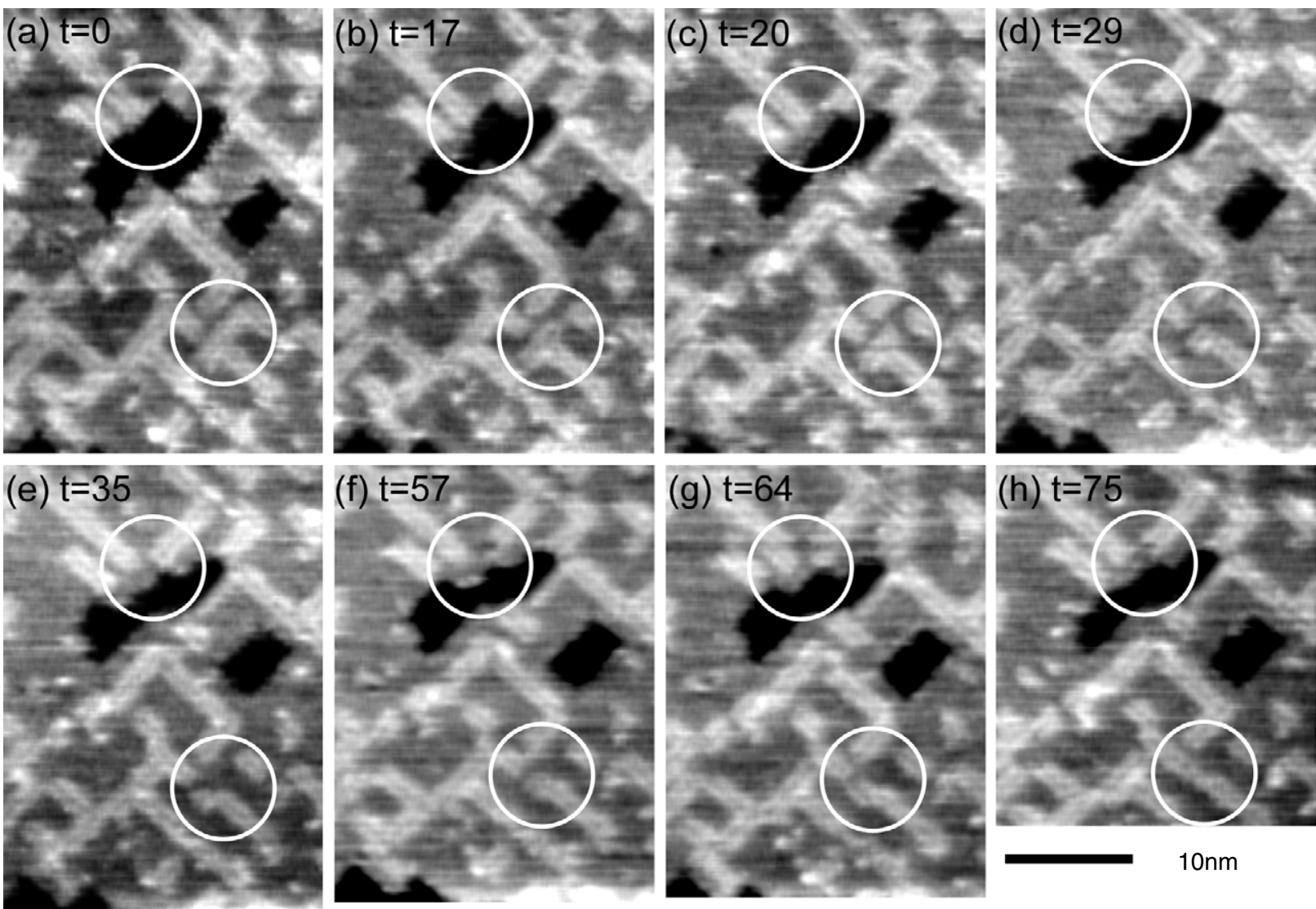

Figure 3. (a)-(h) Growth and dissolution of dilines at $825^{\circ} \mathrm{C}$. Time, $t$, is measured in minutes. The top ring shows the growth of a diline onto a new area of terrace over the course of images (a)-(e). In the lower ring, a nanoline disappears between images (a) and (e). A new nanoline grows perpendicularly in its place during (f)-(h). Sample voltages and tunnelling currents used: (a)-(h) $2.00 \mathrm{~V}, 0.08 \mathrm{nA}$. The supplementary data shows a movie sequence of this series of images (available at stacks.iop.org/Nano/17/3543).

to form with a length of either one or two pairs of atomic protrusions. At room temperature, single and double pairs of atomic protrusions are rarely seen [6].

Our most important observation is that dilines routinely shrink as well as grow, and on rare occasions dissolve entirely. A diline nucleating adjacent to another diline was not observed in the course of these experiments. This suggests that there is no preferential nucleation of dilines adjacent to other dilines. However, once a diline has formed next to another diline, this pair is more stable and grows longer than single dilines. This implies that the stability of domains is greater than that of randomly distributed lines. Taken together, these observations suggest that dilines nucleate randomly and subsequently reorder at elevated temperature to form large, more stable domains.

The thermodynamic evolution of the dilines progresses from the isolated lines to the equilibrium state of ordered domains. However, during the series of images taken in these experiments, the main observations are due to statistical fluctuations. This is because of the short periods of observation relative to the timescale of the evolution of the domains at $825^{\circ} \mathrm{C}$. Thus the movements that we see do not, on the whole, represent a progression towards equilibrium. Using an Arrhenius relationship, we have calculated a value of $1.2 \pm$ $0.1 \mathrm{eV}$ for the activation energy of diffusion of the $\mathrm{TiO}_{x}$ diline units. This value is likely to be a high estimate, as some of the diffusion activity is not observed due to the relatively slow STM scan speed and low resolution of the images.

Trilines and waffles structures have been observed to grow from diline domains. The trilines showed stability when bounded on both sides by dilines, other domains or terrace edges. The waffle structure that we have observed on the $\mathrm{SrTiO}_{3}(001)$ surface is similar to the cross-linked $(1 \times 2)$ surface that forms on the $\mathrm{TiO}_{2}$ surface [13-15]. The theoretical models that have been suggested for the crosslinked $\mathrm{TiO}_{2}$ structure could present a very good starting point for the development of a structural model of the $\mathrm{SrTiO}_{3}$ nanostructures.

The mobility of terrace edges of the $\mathrm{c}(4 \times 2)$ reconstruction at over $800{ }^{\circ} \mathrm{C}$ is also observed, and terraces frequently grow and retreat during the course of imaging. Following annealing for four or five days, however, the terraces become more stable, and fewer changes occur. This mobility of the terrace edges is similar to that of terrace edges on the $\mathrm{SrTiO}_{3}(001)(2 \times 2)$ reconstruction previously observed [10].

These vacuum anneals do not conserve material on the $\mathrm{SrTiO}_{3}$ surface. From Auger studies, there is evidence of $\mathrm{O}$ depletion in $\mathrm{SrTiO}_{3}$ samples annealed at these temperatures in UHV and also an enrichment of $\mathrm{TiO}_{x}$ at the surface. However, one monolayer of $\mathrm{TiO}_{2}$ added to the surface only causes a 

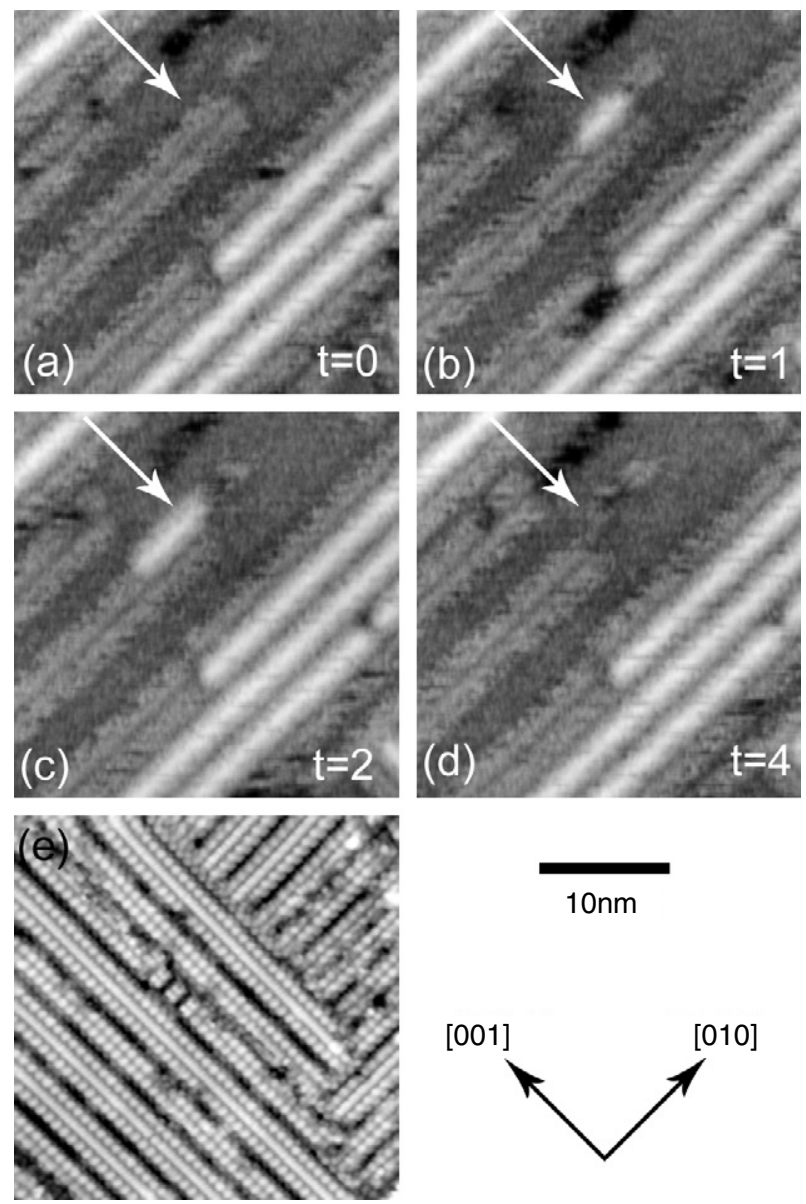

Figure 4. (a)-(d) Formation of a triline from a diline at $850{ }^{\circ} \mathrm{C}$ Time, $t$, is measured in minutes. A pair of dilines are marked in (a). In (b), a triline has nucleated on part of the left-hand diline (arrowed). This triline is observed to grow (c) and then disappear (d). (e) A room-temperature image of dilines and trilines. Sample voltages and tunnelling currents used: (a)-(d) $2.00 \mathrm{~V}, 0.20 \mathrm{nA}$; (e) $0.80 \mathrm{~V}, 0.30 \mathrm{nA}$.

$0.0001 \%$ increase in the number of titanium vacancies in the bulk. Therefore the bulk of the sample is not measurably strontium enriched. Oxygen vacancies within the bulk of the crystal do not significantly influence the electrical conductivity, since the sample is already n-doped with $0.5 \mathrm{wt} \% \mathrm{Nb}$.

It is important to be able to discount the effect of tipsurface interaction from our observations. In order to do this, a smaller area of surface is scanned several times and then compared to the surrounding area. No changes due to the scanning, and thus the tip-surface interaction, are seen. The formation of nanolines and domains whilst annealing at these temperatures without scanning is another indication that the images are not affected by the tip $[6,12]$.

An interesting effect of the high-temperature imaging reported here is the lower annealing temperature required to produce certain nanostructures. This is believed to be due to the length of the anneals performed in these experiments; typically several days, rather than times of up to an hour, as used in previous experiments $[6,12]$. In practice, these timescales are necessary in order to allow the surface to
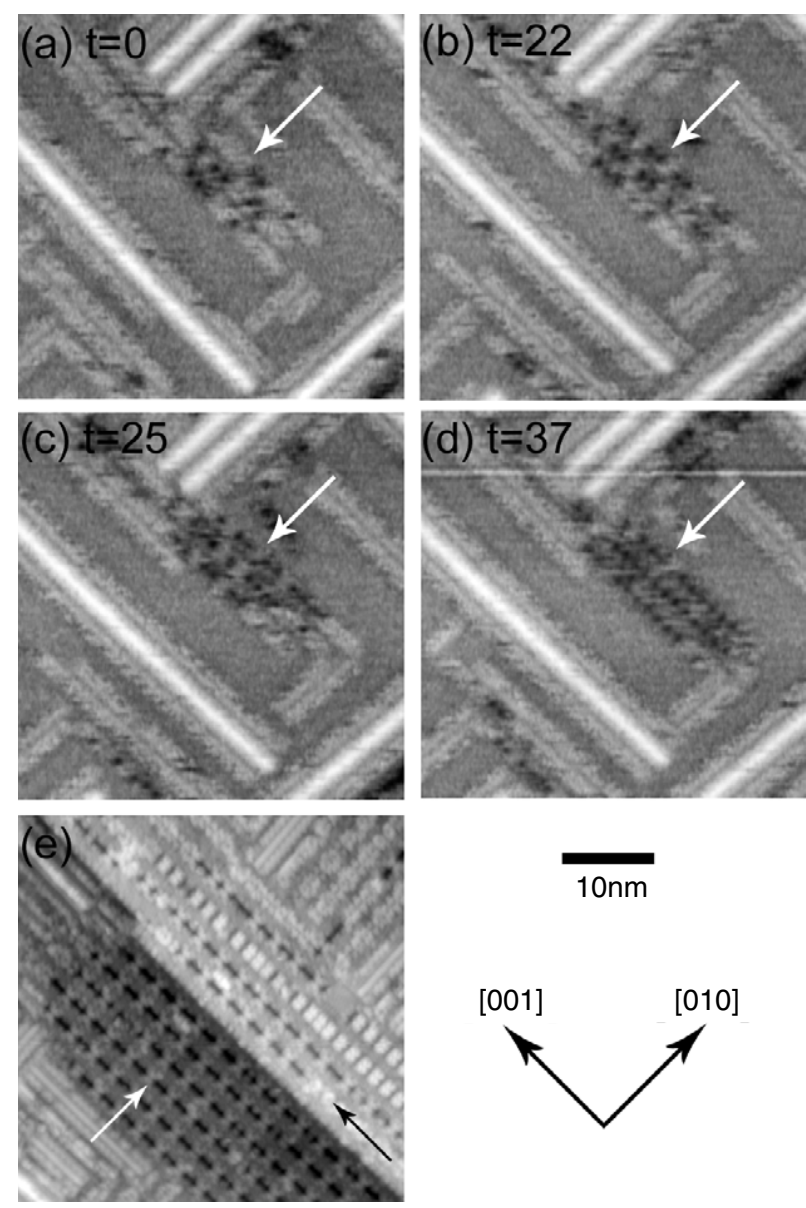

Figure 5. (a)-(d) Formation of a waffle structure from a domain of dilines at $850^{\circ} \mathrm{C}$. Time, $t$, is measured in minutes. In (a), the arrow marks a small domain of dilines, with a few elements of the waffle structure visible within it. In (b) and (c), the amount of waffle structure present increases and, in (d), a large area of waffle structure can be seen. (e) shows a room-temperature image of a large area of waffle structure (white arrow) and a diline which becomes a waffle structure along its length (black arrow). Sample voltages and tunnelling currents used: (a)-(d) $2.00 \mathrm{~V}, 0.20 \mathrm{nA}$; (e) $0.80 \mathrm{~V}$, $0.30 \mathrm{nA}$

equilibriate at the high temperatures used, such that the system is stable enough to image successfully.

\section{Conclusions}

The dynamics of several nanostructures have been observed using hot STM at over $800^{\circ} \mathrm{C}$ for periods of up to $80 \mathrm{~min}$. Observations of the growth of dilines suggests that these randomly nucleate and then reorder into domains at higher temperature. It has also been observed that nanolines are more stable in pairs than when isolated, hence suggesting the stability of domains over randomly distributed lines. The nucleation and growth of both trilines and waffle structures from small diline domains has also been reported.

Overall, this study shows that at high temperatures nanostructures are mobile on the $\mathrm{c}(4 \times 2)$ surface of $\mathrm{SrTiO}_{3}(001)$. It has also demonstrated that the diline domains form by high-temperature reordering of randomly nucleated dilines. 


\section{Acknowledgment}

We acknowledge financial support from the UK Engineering and Physical Sciences Research Council (EPSRC), the Royal Society and the Leverhulme Trust.

\section{References}

[1] Gudiksen M S, Lauhon L J, Wang J, Smith D C and Lieber C M 2002 Nature 415 617-20

[2] Ledentsov N N, Ustinov V M, Shchukin V A, Kop'ev P S, Alferov Z I and Bimberg D 1998 Semiconductors 32 343-65

[3] Beckett P and Jennings A 2002 Proc. 7th Asia-Pacific Computer Systems Architectures Conf. (Melbourne) vol 6 pp 141-50

[4] Nogami J, Liu B Z, Katkov M V, Ohbuchi C and Birge N O 2001 Phys. Rev. B 63 233305-01-4
[5] Chen Y, Ohlberg D A A and Williams R S 2002 J. Appl. Phys. $913213-8$

[6] Castell M R 2002 Surf. Sci. 516 33-42

[7] Iwatsuki M, Sato T and Yamamoto Y 1996 Appl. Surf. Sci. 92 321-30

[8] Harrison B C and Boland J J 2005 Surf. Sci. 594 93-8

[9] Owen J H G, Bowler D R and Miki K 2005 Surf. Sci. 596 163-75

[10] Lippmaa M, Kawasaki M, Ohtomo A, Sato T, Iwatsuki M and Koinuma H 1998 Appl. Surf. Sci. 130-132 582-6

[11] Silly F and Castell M R 2005 J. Phys. Chem. B 109 12316-9

[12] Deak D S, Silly F, Newell D T and Castell M R 2006 J. Phys. Chem. B 110 9246-51

[13] Bennett R A, Stone P, Price N J and Bowker M 1999 Phys. Rev. Lett. 82 3831-4

[14] Smith R D, Bennett R A and Bowker M 2002 Phys. Rev. B 66 035409-1-4

[15] Diebold U 2003 Surf. Sci. Rep. 48 53-229 